\title{
Simultaneous fluorescence and high-resolution bright-field imaging with aberration correction over a wide field-of-view with Fourier ptychographic microscopy (FPM) (Conference Presentation)
}

\author{
Jaebum Chung, Jinho Kim, Xiaoze Ou, Roarke Horstmeyer, Changhuei Yang, California \\ Institute of Technology (United States)
}

\begin{abstract}
We present a method to acquire both fluorescence and high-resolution bright-field images with correction for the spatially varying aberrations over a microscope's wide field-of-view (FOV). First, the procedure applies Fourier ptychographic microscopy (FPM) to retrieve the amplitude and phase of a sample, at a resolution that significantly exceeds the cutoff frequency of the microscope objective lens. At the same time, FPM algorithm is able to leverage on the redundancy within the set of acquired FPM bright-field images to estimate the microscope aberrations, which usually deteriorate in regions further away from the FOV's center. Second, the procedure acquires a raw wide-FOV fluorescence image within the same setup. Lack of moving parts allows us to use the FPM-estimated aberration map to computationally correct for the aberrations in the fluorescence image through deconvolution. Overlaying the aberration-corrected fluorescence image on top of the high-resolution bright-field image can be done with accurate spatial correspondence. This can provide means to identifying fluorescent regions of interest within the context of the sample's bright-field information. An experimental demonstration successfully improves the bright-field resolution of fixed, stained and fluorescently tagged HeLa cells by a factor of 4.9, and reduces the error caused by aberrations in a fluorescence image by $31 \%$, over a field of view of $6.2 \mathrm{~mm}$ by $9.3 \mathrm{~mm}$. For optimal deconvolution, we show the fluorescence image needs to have a signal-to-noise ratio of $\sim 18$.
\end{abstract}

View presentation recording on the SPIE Digital Library:

http://dx.doi.org/10.1117/12.2211974.4848767268001

Three-Dimensional and Multidimensional Microscopy: Image Acquisition and Processing XXIII,

edited by Thomas G. Brown, Carol J. Cogswell, Tony Wilson, Proc. of SPIE Vol. 9713, 97130I

(C) 2016 SPIE · CCC code: 1605-7422/16/\$18 · doi: 10.1117/12.2211974 